\title{
The Antibacterial and Antioxidant Activity of Centella Asiatica Chloroform Extract-loaded Gelatin Nanoparticles
}

\author{
Kittiya Kesornbuakao ${ }^{1}$, Suksun Amornraksa ${ }^{2}$, Malinee Sriariyanun ${ }^{2}$, Suvaluk Asavasanti ${ }^{3}$ and Patchanee Yasurin ${ }^{4}$ \\ ${ }^{1}$ Food Biotechnology Department, Biotechnology Faculty, Assumption University, Bangkok, 10240, Thailand \\ ${ }^{2}$ The Sirindhorn International Thai-German Graduate School of Engineering, King Mongkut's University of Technology North Bangkok \\ ${ }^{3}$ Food Technology \& Engineering Laboratory, Pilot Plant Development \& Training Institute, King Mongkut's University of Technology \\ Thonburi, Bangkok, Thailand \\ ${ }^{4}$ Food Biotechnology Department, Biotechnology Faculty, Assumption University, Bangkok, 10240, Thailand
}

\begin{abstract}
Nanoencapsulation of Centella asaitica (CA) crude chloroform extract seems to be an attractive approach that may improve drug bioavailability and drug delivery system. In the study, CA extract-loaded gelatin nanoparticles (CGNP) were developed by gelatin one-step and two-step desolvation methods, using three different ratios between CA crude chloroform extract and gelatin $(1: 2,1: 3$, and 1:4 w/w). The antibacterial and antioxidants activities of CGNP and CA were compared. The antioxidant activity of CGNP and CA was evaluated by using DPPH radical scavenging assay and ferric reducing antioxidant power (FRAP) assay. Results showed an enhancement of CGNP antibacterial activity against food borne pathogen. The highest inhibition diameters and FRAP determined from CGNP prepared by one-step at 1:4 ratios $(1.03 \pm 0.39 \mathrm{~cm}$ and $1.23 \pm 019 \mathrm{mmol} \mathrm{Fe} 2+/ \mathrm{mg}$ dried weight, respectively). However, CGNP was no significant difference in DPPH radical scavenging activity compared to CA. These results provide useful information for developing effective nanoencapsulation of $\mathrm{CA}$ as effective natural ingredient.
\end{abstract}

\section{Introduction}

Centella asiatica (CA) has been introduced into the group of medicinal plants since the ancient period. The region of CA is located in the tropical and subtropical areas mostly in South East Asia and spread through Western part of the earth. It generally consists of various chemical compositions which have ability to cure many symptoms and diseases related to bacterial infection are involved in the roots and leaves [1]. CA is known that herbs are rich in phenolic compounds [2] that have antioxidant properties as protective agent against free radical compound by scavenging or trapping methods $[3,4]$. Furthermore, CA could inhibited the growth of Bacillus cereus and Listeria monocytogenes at normal, osmotic stress, and high acidic conditions, the growth of pathogenic bacteria in intestines and the growth of both Gram-positive and Gram-negative bacteria[5-8].

In fact, the bioactive compounds such as asiaticoside and asiatica acid can transfer only $50 \%$ or even less than that into the human. That means it has a limitation on the absorption of bioactive compounds from the crude extract of CA when tested under In Vivo and acidic condition. The experiment on In Vitro gave significantly high efficiency on the drug absorption and drug delivery system [9].

Nanotechnology has been used to improve drug bioavailability and drug delivery system. Previous studies of nanoparticles, which are PGLA-nanoparticles, BSA- nanoparticles, and Gelatin-nanoparticles, indicated that nanoparticles technique is applicable to improve bioavailability [10-13]. Gelatin is defined as the hydrophilic proteins derived from collagen extracted from bones, ligaments, skin, and tendons of animal. Gelatin, known as readily and economical source, which is one of several available materials used for generating the nanoparticles, it's acts as the carrier and primary protection for the medicine to be able to increase bioavailability activity.

This research aims to compare antibacterial and antioxidants activity of CA and CA chloroform extractloaded gelatin nanoparticle (CGNP) prepared by gelatin one-step desolvation method and two-step desolvation methods on three different ratios between CA and gelatin $(1: 2,1: 3$, and $1: 4 \mathrm{w} / \mathrm{w})$.

\section{Materials and methods}

\subsection{Preparation of CA}

Fresh CA was purchased from Bangkapi markets in Bangkok, Thailand. The aerial part of CA was used. Fresh CA were washed with tap water and cut into small pieces. Then it was air dried in oven (Memmert UM500) at $45^{\circ} \mathrm{C}$. The dried samples were finely ground into powder. The powder was kept at $4^{\circ} \mathrm{C}$ before used [8]. 


\subsection{Preparation of CA crude chloroform extract}

CA powder is extracted with chloroform using 1:10 ratio $(\mathrm{g} / \mathrm{ml})$. The mixtures are macerated at room temperature, $120 \mathrm{rpm}$, for 48 hours and then are filtered using whatman filter paper no.4. The CA extracts was evaporated at $45^{\circ} \mathrm{C}$ by rotary evaporators (BUCHI Rotavapor R-205). The crude was stored at $-20^{\circ} \mathrm{C}$ prior to use in preparation of CGNP [8].

\subsection{Preparation of Gelatin one-step desolvation nanoparticles}

Gelatin was prepared under constant heat and $\mathrm{pH}$ at $40 \pm 1$ ${ }^{\circ} \mathrm{C}$, pH 3 (adjusting by $0.1 \mathrm{M} \mathrm{HCl}$ ) by dissolving $600 \mathrm{mg}$ of gelatin in $30 \mathrm{ml}$ sterile distilled water. The gelatin nanoparticles were formed after adding CA at different ratio. The ratio between $C A$ and gelatin were $1: 2,1: 3$, and 1:4 w/w. Then, adding $30 \mathrm{ml}$ acetone dropwise. 100 $\mu \mathrm{L} 8 \% \mathrm{v} / \mathrm{v}$ glutaraldehyde solution was added to stabilize of CGNP and the solution was stirred gently for 2 hours. CGNP were centrifuged and washed with distilled water. The centrifuged particles were resuspended and disperse in 3\% mannitol, then freezedried for 48 hours. The dried CGNP were kept $4^{\circ} \mathrm{C}$ prior to use in antioxidant assay [13].

\subsection{Preparation of Gelatin two-step desolvation nanoparticles}

The encapsulation of CA in gelatin nanoparticles was modified from Azimi et al. [13]. $600 \mathrm{mg}$ gelatin was added in $30 \mathrm{ml}$ sterile distilled water under constant temperature at $40 \pm 1{ }^{\circ} \mathrm{C}$. The precipitation of high molecular weight gelatin was obtained by adding $30 \mathrm{ml}$ acetone into gelatin solution. After 15 minutes, the high molecular weight gelatin was redissolved with $30 \mathrm{ml}$ sterile distilled water at $40 \pm 1^{\circ} \mathrm{C}, \mathrm{pH} 3$ and stirred gently. CGNP was formed by adding $\mathrm{CA}$ at different ratios. The ratios between $C A$ and gelatin were $1: 2,1: 3$, and 1:4 w/w. Then, adding $30 \mathrm{ml}$ acetone dropwise. The stabilization of CGNP was generated by adding $100 \mu \mathrm{L}$ glutaraldehyde solution $(8 \% \mathrm{v} / \mathrm{v})$ and stirring gently for 2 hours. CGNP were centrifuged and washed with distilled water. The centrifuged particles were resuspended and disperse in 3\% mannitol, then freeze-dried for 48 hours. The dried CGNP were kept $4^{\circ} \mathrm{C}$ prior to use in antioxidant assay.

\subsection{Antimicrobial activity}

CA and CGNP were added on MHA plates which were swabbed with $100 \mu \mathrm{L}$ of bacterial cultures (approx. $1.5 \times 108 \mathrm{CFU} / \mathrm{ml}$ ) was referred to the modified agar well diffusion method adapted from Rattanakom and Yasurin [8]. Penicillin G and DMSO were used as positive and negative controls, respectively. After 24 hours of incubation, the inhibition zone were measured to determine how effective CA and CGNP could inhibit certain microorganisms which were Escherichia coli ATCC25822, Bacillus cereus, B. subtilis, Staphylococcus aureus, Salmonella enterica Typhimurium U302 (DT104b), S. enterica Enteritidis (human), and S. enterica 4,5,12:i:- (human) US clone.

\subsection{Antioxidant activity by Ferric reducing antioxidant power (FRAP) assay}

The modified ferric reducing antioxidant potential assay [14] was used to determine FRAP value of CA and CGNP. The FRAP reagent was prepared using $300 \mathrm{mmol}$ sodium acetate buffer at $\mathrm{pH} 3.6,20 \mathrm{mmol}$ iron chloride and $10 \mathrm{mmol}$ 2,4,6-tripyridyl-s-triazine dissolved in 40 mmol hydrochloric acid at a ratio of 10:1:1 (v:v:v). The reagent was incubated at $37^{\circ} \mathrm{C}$ for 10 minutes before use. The $20 \mu 1$ of $1 \mathrm{mg} / \mathrm{ml}$ of CA and CGNP was added, followed by adding $1000 \mu \mathrm{l}$ of FRAP reagent vigorously and kept in the dark for 30 minutes. The optical density (OD) of this mixture was measured at $593 \mathrm{~nm}$. FRAP values were expressed as mmol $\mathrm{Fe} 2+/ \mathrm{mg}$ of sample. All measurements were done in triplicate and three replications independently.

\subsection{Antioxidant activity by 2,2-Diphenyl-1- picrylhydrazil (DPPH) radical scavenging activity}

This method is modified from Brand-Williams [15] to determine the antioxidant activities of CA and CGNP. The mixture of $100 \mu \mathrm{L}$ CA or CGNP and $3.9 \mathrm{~mL}$ of methanol DPPH solution ( $5 \times 10-5 \mathrm{~mol} / \mathrm{L})$ is shaken thoroughly and kept in the dark room for 30 minutes. The mixture is measured by UV-vis spectrophotometer at 517 $\mathrm{nm}$. The unit of $\mu \mathrm{g} / \mathrm{mL}$ of gallic acid equivalent (GAE) per $1 \mathrm{~g}$ sample is used to express results.

\subsection{Statistical analysis}

All experiments were conducted in three replications and statistical analysis was accomplished using ANOVA with Duncan's multiple range tests $(\mathrm{p}<0.05)$ by SAS software version 9.4.

\section{Results and discussion}

\subsection{Antioxidant activities}

As the antioxidant activity of CA come from the complex of phytochemicals and involvement of many reactions, the measurement of the activity cannot be done accurately with only one assay. The combination of DPPH radical scavenging activity and Ferric reducing antioxidant power (FRAP) were used to evaluate the antioxidant activity.

The antioxidant activity of all CGNPs and CA were statistically analyzed to see the different between each 
sample that were prepared from different preparation methods and ratios. The results from Figure 1 and Figure 2 demonstrated that CGNP prepared by one-step desolvations method showed significantly greater FRAP than both CA and CGNP two-step at all ratios $(\mathrm{p}<0.05)$.

FRAP

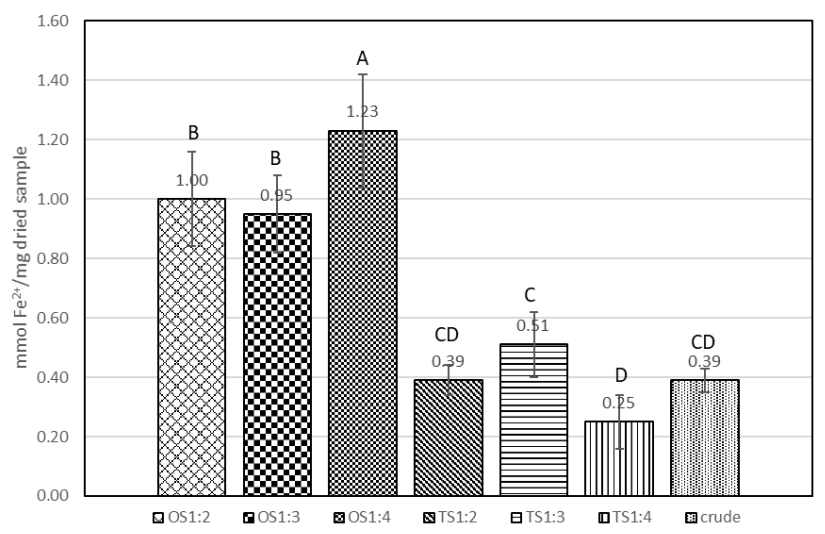

Figure 1. Antioxidant activity by Ferric reducing antioxidant power (FRAP) assay of CA and CGNP prepared at different ratios between CA and Gelatin. OS and TS represented CGNP one-step and CGNP two-step respectively. A, B represented significantly different value in a column at $\mathrm{p}<0.05$.

DPPH

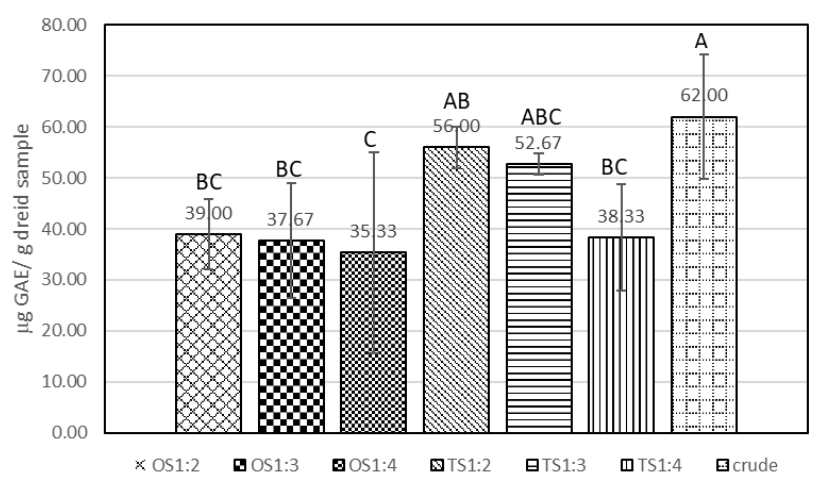

Figure 2. Antioxidant activity by 2, 2-Diphenyl-1picrylhydrazil (DPPH) radical scavenging activity of CA and CGNP prepared at different ratios between CA and Gelatin. OS and TS represented CGNP one-step and CGNP two-step respectively. A, B represented significantly different value in a column at $\mathrm{p}<0.05$.

According to FRAP assay, determination of ability to reduce of ferric iron $\left(\mathrm{Fe}^{3+}\right)$ to ferrous iron $\left(\mathrm{Fe}^{2+}\right)$ [16], $\mathrm{CA}$ nanoencapsulated could enhance FRAP of CA. In addition, CGNP one-step nanoencapsulation structure may differ from two-step due to size of the protein and effect to FRAP values by releasing of CA. Moreover, CGNP prepared by one-step desolvation method at ratio 1:4 showed highest FRAP $\left(1.23 \pm 019 \mathrm{mmol} \mathrm{Fe}^{2+} / \mathrm{mg}\right.$ dried weight) among CGNP. However, CGNP one-step at all ratios exhibited significantly lower in DPPH radical scavenging activity than CA $(\mathrm{p}<0.05)$. DPPH radical scavenging assay measures the reducing ability of antioxidants toward DPPH [16]. The decrease in antioxidant activity of nanoencapsulated active compounds is probably related to the protectability of the droplets in the system that slow down the reaction rate of reduction [17]. In contrast, CGNP prepared with two-step desolvation method at ratio $1: 2$ and 1:3 showed no significantly different in DPPH radical scavenging activity compared with CA $(56.00 \pm 4.00$ and $52.67 \pm$ $2.08 \mathrm{mmol} \mathrm{Fe}^{2+} / \mathrm{mg}$ dried weight, respectively).

\subsection{Antibacterials}

CGNPs were prepare by one-step and two-step gelatin desolvation methods[13]. Both CGNP preparation were done with different ratio of CA to gelatin at 1:2,1:3, and 1:4. The well agar diffusion method was used for evaluating antibacterial activity of CGNPs and CA with different concentration $(100,200$, and $300 \mu \mathrm{l} / \mathrm{ml})$ against seven food borne pathogens (Escherichia coli ATCC25822, Streptococcus aureus, Bacillus cereus, B. subtilis, Salmonella enterica Typhimurium U302 (DT104b), S. enterica Enteritidis (human), and $S$. enterica 4,5,12:i:- (human) US clone).

The results showed that antibacterial activity of CGNP were clearly increased on S.s aureus, S. enterica Typhimurium U302 (DT104b), S. enterica Enteritidis (human), and S. enterica 4,5,12:i:- (human) US clone comparing with CA as shown in Table 1. The CGNP likely more affected to gram-negative ( $E$. coli ATCC25822, S. enterica Typhimurium U302 (DT104b), S. enterica Enteritidis (human), and $S$. enterica 4, 5, 12: i: - (human) US clone) than gram-positive bacteria ( $B$. cereus, B. subtilis, and $S$. aureus). Because the structure of gram-negative and gram-positive bacteria are different. Gram-negative bacteria has inner membrane, thin layer made of peptidoglycan, and outer membrane whereas gram-positive has an inner membrane covered with thick layer of peptidoglycan $[18,19]$. The thick layer of peptidoglycan have high protectaility for gram-positive bacteria which harder for CGNP to get through the cells. In addition, the highest inhibition zone of CGNP was $1.03 \pm 0.39 \mathrm{~cm}$ against $S$. enterica Enteritidis using gelatin one-step desolvation method on ratio $1: 4,300 \mu \mathrm{g} / \mathrm{ml}$.

\section{Conclusion}

Overall, CGNPs exhibited interesting antibacterial and antioxidant activity. The DPPH radical scavenging of CGNP tend to decrease because it could protect an active compound and slow down the oxidative reaction. CGNPs enhance antibacterial activity on human pathogen, especially on gram-negative bacteria. These results provide a promising for improving bioavailability of CA

\section{Acknowledgements}

This work was supported by the research grant of Research and Researchers for Industries (RRI) by Thailand Research Fund (TRF) (PHD59I0074) and King Mongkut's University of Technology, North Bangkok 
(Research University Grant No. KMUTNB-61-GOV-01$55)$.

\section{References}

1. A. J. Das, J. Biol. Act. Prod. Nat., 1:4 (2011)

2. P. Yasurin, M. Sriariyanun, T. Phusantisampan, KMUTNB Int. J. Appl. Sci. Technol., 9 (2016)

3. D de Beer, E. Jouber, W.C.A. Gelderblom, and M. Manley, South African Journal of Enology \& Viticulture, 23 (2002)

4. D. Huang, B. Ou, and R. L.Prior, J. Agric. Food Chem., 53 (2005)

5. N. Pitinidhipat and P. Yasurin, AU J.T., 15 (2012)

6. C. V. Utami, N. Pitinidhipat, and P. Yasurin, KMITL Sci. Tech. J., 12 (2012)

7. B. Mamtha, K. Kavitha, K. K. Srinivasan, and P. G. Shivananda, Indian J. Pharmacol, 36 (2004)

8. S. Rattanakom and P. Yasurin, Orient. J. Chem., 34 (2015)

9. W. Kriengsinyos, C. Chitchumroonchokchai, and P. Siriprapa, FASEB Journal, 20 (2006)

10. X. Xie, Q. Tao, Y. Zou, F. Zhang, M. Guo, Y. Wang, H. Wang, Q. Zhou, and S. Yu, J. Agric. Food. Chem., 59 (2011)

11. Y. Zhan, Y. Min, Z. Zhang, G. Hong, and Q. Xiong, anoscale Res. Lett., 9 (2014)

12. K. Kesornbuakao, and P. Yasurin, Oreintal Journal of Chemistry., 32 (2016)

13. B. Azimi, P. Nourpanah, M. Rabiee, and S. Arbab, Iran. Biom. J., 18 (2014)

14. IF. Benzie, and JJ. Strain, Methods Enzymol., 299 (1999)

15. W. Brand-Williams, M. E. Cuvelier, and C. Berset, LWT - Food Science and Technology, 28 (1995)

16. K.D.R.R. Silva, and M.S.F. Sirasa, Food Chemistry, 238 (2016)

17. M. B. Jemaa, H. Falleh, R. Serairi, M. A. Neves, M. Snoussi, H. Isoda, M. Nakajima, and R. Ksouri, Innovative Food Science \& Emerging Technologies., 45 (2018)

18. M. Madigan and J. Martinko, 11th ed., Englewood Cliffs. NJ: Prentice Hall, (2005)

19. L. Brown, J.M. Wolf, R. Prados-Rosales, and A. Casadevall, Nat. Rev. Microbiol., 13 (2015) 


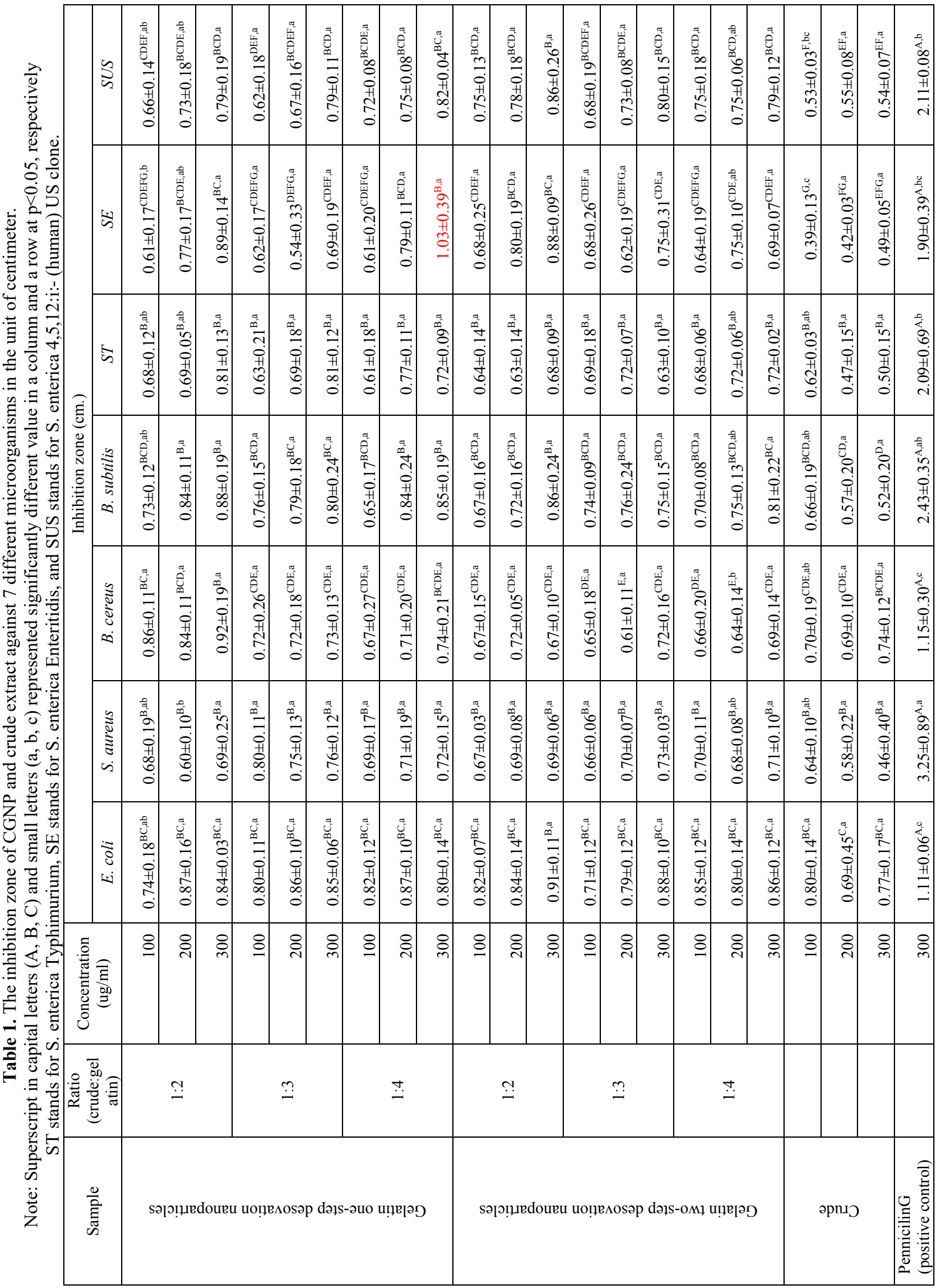




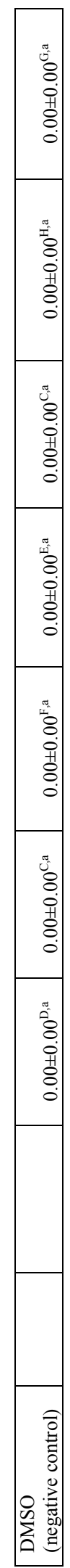

